\title{
Economic Policy Uncertainty and Chinese Stock Market Volatility: A CARR-MIDAS Approach
}

\author{
Xinyu Wu $\mathbb{D}^{1}{ }^{1}$ Tianyu Liu, ${ }^{1}$ and Haibin $X^{2}{ }^{2}$ \\ ${ }^{1}$ School of Finance, Anhui University of Finance and Economics, Bengbu 233030, China \\ ${ }^{2}$ School of Banking and Finance, University of International Business and Economics, Beijing 100029, China \\ Correspondence should be addressed to Xinyu Wu; xywu@aufe.edu.cn
}

Received 21 April 2021; Accepted 18 September 2021; Published 30 September 2021

Academic Editor: Zakia Hammouch

Copyright (c) 2021 Xinyu Wu et al. This is an open access article distributed under the Creative Commons Attribution License, which permits unrestricted use, distribution, and reproduction in any medium, provided the original work is properly cited.

Intraday range (the difference between intraday high and low prices) is often used to measure volatility, which has proven to be a more efficient volatility estimator than the return-based one. Meanwhile, a growing body of studies has found that economic policy uncertainty (EPU) has important impact on stock market volatility. In this paper, building on the range-based volatility model, namely, the conditional autoregressive range (CARR) model, we introduce the CARR-mixed-data sampling (CARRMIDAS) model framework by considering intraday information to investigate the impact of EPU on the volatility of Chinese stock market and to explore the predictive ability of EPU for Chinese stock market. The empirical results show that both the China EPU (CEPU) and global EPU (GEPU) have a significantly negative effect on the long-run volatility of Chinese stock market. Furthermore, we find that taking into account the CEPU and GEPU leads to substantial improvement in the ability to forecast the volatility of Chinese stock market. We also find that the CEPU provides superior volatility forecasts compared to the GEPU. Our findings are robust to different forecasting windows.

\section{Introduction}

Modelling and forecasting volatility is of great importance for many financial applications such as asset allocation, risk measurement, and option pricing. It is well known that stock market volatility exhibits clustering and high persistence. Although predictable, it is still difficult to forecast accurately. In this paper, we investigate the impact of economic policy uncertainty (EPU) on the volatility of Chinese stock market and explore the predictive ability of EPU for Chinese stock market. By doing so, we shed new light on the role of EPU in Chinese stock market volatility.

Recently, Baker et al. [1] developed an EPU index which is based on newspaper coverage to measure policy-related economic uncertainty, and it has received a great deal of attention in the literature (see Al-Thaqeb and Algharabali [2] for a detailed literature review). As measured by the EPU index of Baker et al. [1], the level of EPU keeps rising since the global financial crisis of 2008. In particular, the current level of EPU is at extremely elevated levels due to a series of events including the US-China trade war and the coronavirus (COVID-19) pandemic, which have forced governments around the world to make frequent changes to their policies in order to limit the economic impact of these events. Intuitively, this high EPU may affect investors' investment decisions and hence stock markets. Indeed, recent studies have shown that EPU has a significant impact on stock markets. Pástor and Veronesi $[3,4]$ investigated the impact of EPU and political uncertainty on the volatility of US stock market and observed that policy changes increase volatility, risk premia, and correlations among stocks. Liu and Zhang [5] found that incorporating EPU as an additional predictive variable into the HAR-RV model significantly improves the forecasting ability of the model for the volatility of US stock market. Arouri et al. [6] investigated the impact of EPU on the US stock market returns and found that an increase in EPU significantly decreases stock returns and this effect is stronger and persistent during extreme volatility periods. Liu et al. [7] found that EPU has a significant impact on the volatility of the US stock market. 
Moreover, out-of-sample results show that adding EPU as explanatory variable to the volatility model can indeed improve its forecasting performance. Using the VAR model, Tsai [8] analyzed the impact of EPU on the stock returns of 22 stock markets worldwide. Duan et al. [9] investigated the impacts of leverage effect and EPU on future volatility in the framework of regime switching. Mei et al. [10] found that taking into account the US EPU leads to substantial improvement in the ability to forecast the volatility of European stock market. Xiong et al. [11] studied the correlation between China EPU (CEPU) and Chinese stock market returns. Yu and Song [12] found that global EPU (GEPU) has a significant impact on the volatility of US stock market. Balcilar et al. [13] showed that the EPU of major economies is likely to predict stock market volatility in emerging stock markets. Chiang [14] examined EPU and risks on excess stock returns for G7 markets using monthly observations and found that the stock returns are negatively correlated with EPU innovation. However, those studies mainly focus on the US and European stock markets.

To our knowledge, there are few studies investigating the impact of EPU on the volatility of Chinese stock market. Chinese stock market is one of the largest and most important emerging stock markets, which has gained a great deal of attention from investors. It is characterized by large fluctuations, and it is expected that it may be affected by changes in economic policies. Naturally, investigation of the impact of EPU on the volatility of Chinese stock market and exploration of the predictive ability of EPU are important for investors in Chinese stock market, who are eager to understand the dynamics of volatility for the sake of portfolio allocation and risk management.

Previous studies investigated the impact of EPU on the volatility of Chinese stock market relying mainly on the generalized autoregressive conditional heteroskedasticitymixed-data sampling (GARCH-MIDAS) model by Engle et al. [15]. The choice of MIDAS-based approach is based on the fact that the EPU and stock market data are sampled at different frequencies, i.e., EPU is on a monthly basis, while stock market data are available at a daily frequency. Using the GARCH-MIDAS approach, Yu et al. [16] and $\mathrm{Yu}$ and Huang [17] provided evidence that GEPU has a significantly positive effect on the volatility of Chinese stock market and has predictive ability for the volatility of Chinese stock market, while Li et al. [18] explored the effects of directional (up and down) GEPU on Chinese stock market volatility and provided evidence that up and down GEPU have positive impacts on Chinese stock market volatility and the GEPU index predicts Chinese stock market volatility. Furthermore, Wang et al. [19] employed a GARCH-MIDAS model with skew Student's $t$-distribution to examine the impact of domestic and foreign EPU on the volatility of China's financial stocks and found that the EPU index has a negative impact on the volatility of China's financial stocks. Recently, Li et al. [20] used a predictive regression approach to investigate the impact of EPU on Chinese stock market volatility and provided evidence that the EPU index has a significantly negative impact on future volatility of Chinese stock market. The predictive regression model adopted by $\mathrm{Li}$ et al. [20] requires the frequency of EPU to match that of predicted (realized) volatility. As a result, Li et al. [20] employed the monthly data to perform the analysis, which may ignore important structural features of stock market data and constrain the choice of forecast horizons (i.e., one month).

The GARCH-MIDAS model offers a convenient framework to combine data that are sampled at different frequencies, avoiding the loss of information. Essentially, the GARCH-MIDAS model is a return-based volatility model that uses daily (squared or absolute) close-to-close returns to estimate volatility, which fails to exploit the intraday information. An alternative approach for estimating volatility is to apply the intraday range, which is based on intraday high and low prices. The main advantage of the range-based volatility estimator over the traditional volatility estimator based on closing prices (or close-to-close returns) is its information content: the range contains information about all intraday price movements. Parkinson [21] showed that the range is a more accurate volatility estimator than the volatility estimator based on the daily returns. Since then, the range-based volatility has received a great deal of attention in the literature (see, e.g., Alizadeh et al. [22], Brandt and Jones [23], Li and Hong [24], Degiannakis and Livada [25], and Chou et al. [26]). In particular, Chou [27] introduced a conditional autoregressive range (CARR) model to model the dynamics of the range, showing that the model yields more accurate volatility estimates than the traditional return-based GARCH model.

Motivated by the above interpretation, in this paper, we aim to introduce the CARR-MIDAS approach to investigate the impact of EPU on the volatility of Chinese stock market and to explore the predictive ability of EPU for Chinese stock market. Importantly, our methodology is founded on the range-based CARR model, which allows us to exploit intraday information. Also, the proposed CARR-MIDAS model builds upon the GARCH-MIDAS model, which features a multiplicative decomposition of volatility into a short-run (high-frequency) component and a long-run (low-frequency) component that allows us to incorporate exogenous explanatory variables such as the EPU. It is claimed that the multiplicative component model is able to capture complex volatility dynamics such as the high persistence of volatility as well as to handle well structural breaks or nonstationarities in volatility (see Wang and Ghysels [28], Conrad and Kleen [29], and Xie [30]).

The contributions of the paper are twofold. First, we introduce a new range-based CARR-MIDAS model framework combing the insights of range-based CARR and return-based GARCH-MIDAS. Compared to the CARR model, the CARR-MIDAS model allows us to incorporate exogenous explanatory variables directly into the long-run component of volatility. Hence, using the CARR-MIDAS model that incorporates EPU, we are able to study the relation between EPU and stock market volatility. Compared to the return-based GARCH-MIDAS model, our proposed range-based CARR-MIDAS model utilizes more (intraday) information for estimating volatility. 
Second, using the CARR-MIDAS approach, an empirical analysis based on the Shanghai Stock Exchange Composite Index (SSEC) of China and two EPU indices, namely, the CEPU and GEPU, is conducted. The empirical results show that the CARR-MIDAS models (with the CEPU and GEPU) outperform the CARR model in terms of both in-sample fitting and out-of-sample forecast. Both the CEPU and GEPU have a significantly negative impact on the Chinese stock market volatility. Moreover, both the CEPU and GEPU can greatly improve the forecast accuracy for Chinese stock market volatility for forecast horizons of one-day ahead up to one month. Most importantly, we find that the CEPU provides superior volatility forecasts compared to the GEPU. Our empirical results are robust to different forecasting windows.

The remainder of the paper is organized as follows. In Section 2, we introduce the methodology of our CARRMIDAS model. Section 3 presents the empirical results, while Section 4 concludes the paper.

\section{Methodology}

2.1. The Range. In this paper, we employ the intraday range by considering intraday information to investigate the volatility of Chinese stock market. The intraday range of Parkinson [21] is defined as

$$
R_{i, t}=\frac{\log \left(H_{i, t}\right)-\log \left(L_{i, t}\right)}{\sqrt{4 \log (2)}}
$$

where $H_{i, t}$ and $L_{i, t}$ are the highest and lowest prices of an asset observed on day $i$ in month $t$, respectively. Parkinson [21] showed that the intraday range given by equation (1) is an unbiased estimator of volatility. Assuming that the logprice of an asset follows a Brownian motion with zero drift, Parkinson [21] proved that the range is 4.9 times more efficient than the daily squared return based on the closing prices. Moreover, Degiannakis and Livada [25] showed that the price range volatility estimator is more accurate compared to the realized volatility estimator constructed from five, or less, intraday returns.

2.2. The CARR-MIDAS Model. Inspired by the return-based GARCH-MIDAS model of Engle et al. [15], we propose in the paper the range-based CARR-MIDAS model to describe the dynamics of the range, which can be written as

$$
\begin{aligned}
R_{i, t} & =\lambda_{i, t} \varepsilon_{i, t}, \quad \varepsilon_{i, t} \mid \mathscr{F}_{i-1, t} \sim \text { i.i.d. } f(\cdot), \\
\lambda_{i, t} & =\tau_{t} g_{i, t}, \\
g_{i, t} & =(1-\alpha-\beta)+\alpha \frac{R_{i-1, t}}{\tau_{t}}+\beta g_{i-1, t}, \\
\log \left(\tau_{t}\right) & =m+\theta_{1} \sum_{k=1}^{K} \varphi_{k}\left(\gamma_{1}\right) \log \left(R R V_{t-k}\right),
\end{aligned}
$$

where $\lambda_{i, t}$ is the conditional mean of the range based on the information set, $\mathscr{F}_{i-1, t}$, up to day $i-1$ of month $t$, the error term $\varepsilon_{i, t}$ is assumed to be independent and identically distributed (i.i.d.) with unit mean from density $f(\cdot)$ with positive support, and $\mathrm{RRV}_{t}=\sum_{i=1}^{N_{t}} R_{i, t}^{2}$ is the realized range volatility (RRV) in month $t$, where $N_{t}$ is the number of trading days in month $t$. Note that the conditional range, $\lambda_{i, t}$, is multiplicatively decomposed into a short-run (highfrequency) component, $g_{i, t}$, and a long-run (low-frequency) component, $\tau_{t}$. The short-run component, $g_{i, t}$, is specified as a $\operatorname{GARCH}(1,1)$-like process. To ensure nonnegativity and stationarity for the short-run component $g_{i, t}$, we assume that $\alpha>0, \beta>0$, and $\alpha+\beta<1$. The long-run component, $\tau_{t}$, is modelled in the spirit of the MIDAS regression, which is driven by the smoothing monthly RRV with the weighting scheme $\varphi_{k}$. One-parameter beta polynomial is employed as the weighting scheme due to its parsimony and flexibility:

$$
\varphi_{k}(\gamma)=\frac{(1-k / K)^{\gamma-1}}{\sum_{j=1}^{K}(1-j / K)^{\gamma-1}},
$$

where $K$ is the number of MIDAS lags with $\sum_{k=1}^{K} \varphi_{k}(\gamma)=1$.

The model specification described above is the standard CARR-MIDAS model, which is more flexible than the original CARR model of Chou [27]. It is straightforward to show that

$$
\lambda_{i, t}=\omega_{t}+\alpha R_{i-1, t}+\beta \lambda_{i-1, t},
$$

where $\omega_{t}=(1-\alpha-\beta) \tau_{t}$ implies the time-varying parameter which allows to capture structural changes in conditional volatility. Lamoureux and Lastrapes [31] showed that structural changes should be taken into account when modelling volatility, otherwise may cause spurious apparent persistence in the volatility process (or long-memory volatility). Assuming constant long-run component, the CARR-MIDAS model reduces to the CARR model:

$$
\begin{aligned}
R_{i, t} & =\lambda_{i, t} \varepsilon_{i, t}, \quad \varepsilon_{i, t} \mid \mathscr{F}_{i-1, t} \sim \text { i.i.d. } f(\cdot), \\
\lambda_{i, t} & =\omega+\alpha R_{i-1, t}+\beta \lambda_{i-1, t},
\end{aligned}
$$

where $\omega=(1-\alpha-\beta) \tau$.

To further explore the explanatory ability of EPU on Chinese stock market volatility, we extend the long-run component in equation (2) by incorporating EPU as

$$
\begin{aligned}
\log \left(\tau_{t}\right)= & m+\theta_{1} \sum_{k=1}^{K} \varphi_{k}\left(\gamma_{1}\right) \log \left(\operatorname{RRV}_{t-k}\right) \\
& +\theta_{2} \sum_{k=1}^{K} \varphi_{k}\left(\gamma_{2}\right) \log \left(\mathrm{EPU}_{t-k}\right),
\end{aligned}
$$

where $\mathrm{EPU}_{t}$ is the EPU index in month $t$. In regard to the EPU index, we consider in this paper the CEPU and GEPU.

2.3. Maximum Likelihood Estimation. The CARR-MIDAS model is easy to estimate. We use the quasimaximum likelihood method to estimate the parameters of the CARRMIDAS model. The log-likelihood function of the CARRMIDAS model can be written as 


$$
\ell(\Theta)=-\sum_{t=1}^{T} \sum_{i=1}^{N_{t}}\left[\log \left(\lambda_{i, t}\right)-\log \left(f\left(\frac{R_{i, t}}{\lambda_{i, t}}\right)\right)\right],
$$

where $\Theta$ is the vector of all model parameters.

To implement the CARR-MIDAS model, we need to specify the density $f(\cdot)$ (or the distribution of the error term $\left.\varepsilon_{i, t}\right)$. We choose the Gamma density function, which can be written as

$$
f\left(\varepsilon_{t}\right)=\frac{\nu}{\Gamma(\nu)}\left(\nu \varepsilon_{i, t}\right)^{\nu-1} \exp \left(-\nu \varepsilon_{i, t}\right), \quad \varepsilon_{i, t} \geq 0,
$$

where $v(v>0)$ is the shape parameter. It is important to note that the Gamma distribution is flexible and includes the exponential distribution as a special case when $v$ takes the value of 1 . Moreover, Xie and $\mathrm{Wu}$ [32] showed that the Gamma distribution can reduce both the inlier and outlier problems.

Then, the log-likelihood function can be written as

$$
\ell(\Theta)=-\sum_{t=1}^{T} \sum_{i=1}^{N_{t}}\left[\log \left(\lambda_{i, t}\right)+\log (\Gamma(\nu))-v \log (\nu)-(\nu-1) \log \left(\frac{R_{i, t}}{\lambda_{i, t}}\right)+\nu \frac{R_{i, t}}{\lambda_{i, t}}\right] .
$$

Hence, the maximum likelihood estimators, $\widehat{\Theta}$, can be obtained by maximizing the log-likelihood function in equation (9):

$$
\widehat{\Theta}=\arg \max _{\Theta} \ell(\Theta) .
$$

\section{Empirical Results}

3.1. Data. We use the CARR-MIDAS approach to investigate the impact of EPU on the volatility of Chinese stock market and to explore the predictive ability of EPU for Chinese stock market. The data used in the paper consist of daily open, high, low, and close prices for the Shanghai Stock Exchange Composite Index (SSEC) of China from January 4, 2005 , to December 31,2020, resulting in a total of 3889 daily observations. The data are obtained from Wind Database of China. The intraday range is computed using equation (1). As EPU, we consider the monthly CEPU index and GEPU (current) index constructed by Baker et al. [1], which are obtained from https://www.policy.uncertainty.com/. The daily range of SSEC and monthly EPU (CEPU and GEPU) are presented in Figure 1, which shows that the well-known behaviors of volatility clustering in the Chinese stock market are apparent, and Chinese stock market experienced particularly strong fluctuations during the periods of global financial crisis of 2007-2008 and Chinese stock market turbulence of 2015-2016. In addition, we find that the EPU indices seem to increase over the sample period particularly in the recent years. Moreover, it is interesting to note that the CEPU is generally larger and significantly more volatile than the GEPU, suggesting that Chinese government makes more frequent adjustments of economic policy.

Table 1 presents summary statistics. Panel A reports summary statistics of the daily range for the SSEC. The results show that the SSEC range is positively skewed and highly leptokurtic. From the Jarque-Bera statistics, we observe that the SSEC range fails the normality assumption. The Ljung-Box Q statistics for autocorrelation up to 10 lags provide evidence of high persistence (or long-memory property) for the SSEC range, suggesting that our proposed
CARR-MIDAS framework that assumes a multiplicative component structure might be appropriate for modelling the conditional range. Panel B reports summary statistics of the monthly CEPU and GEPU. It is worth noting that the CEPU is significantly larger and more volatile than the GEPU, which is consistent with the findings in Figure 1, indicating that economic policy changes more often in China.

3.2. Estimation Results. Table 2 reports the estimation results for the standard CARR-MIDAS model and its specification with the EPU (CARR-MIDAS-CEPU and CARR-MIDASGEPU) discussed in Section 2.2. In addition, estimates for the CARR model are presented for the purpose of comparison. For the CARR-MIDAS specifications, we employ three MIDAS lag years, i.e., we choose $K=36$ for the monthly RRV and EPU. Conrad and Kleen [29] showed that the data will identify the optimal weighting scheme as long as $K$ is chosen reasonably large.

As can be seen from the table, the estimate of the persistence coefficient $\alpha+\beta$ in the CARR model is close to one, showing high persistence in the conditional range process. Note also that, in the CARR-MIDAS estimation results, the estimates of the persistence coefficient of the short-run component, $\alpha+\beta$, are less than one, with its magnitude smaller than that of the CARR, indicating that accounting for long-run component reduces persistence in the short-run component. Additionally, the estimates of the parameter $\theta_{1}$ are significant positive, which suggests that the monthly RRV is positively related to long-run volatility of Chinese stock market. It is interesting to note that the estimates of the parameter $\theta_{2}$ in the CARR-MIDAS-CEPU and CARR-MIDAS-GEPU models are all negative and statistically significant, implying that both CEPU and GEPU have significantly negative effect on long-run volatility of Chinese stock market. That is, an increase in CEPU or GEPU predicts lower levels of long-run volatility of Chinese stock market. This result is consistent with the findings of Li et al. [20] and Wang et al. [19].

Figure 2 plots the conditional range $\left(\lambda_{i, t}\right)$ along with the long-run component $\left(\tau_{t}\right)$ from the CARR-MIDAS 


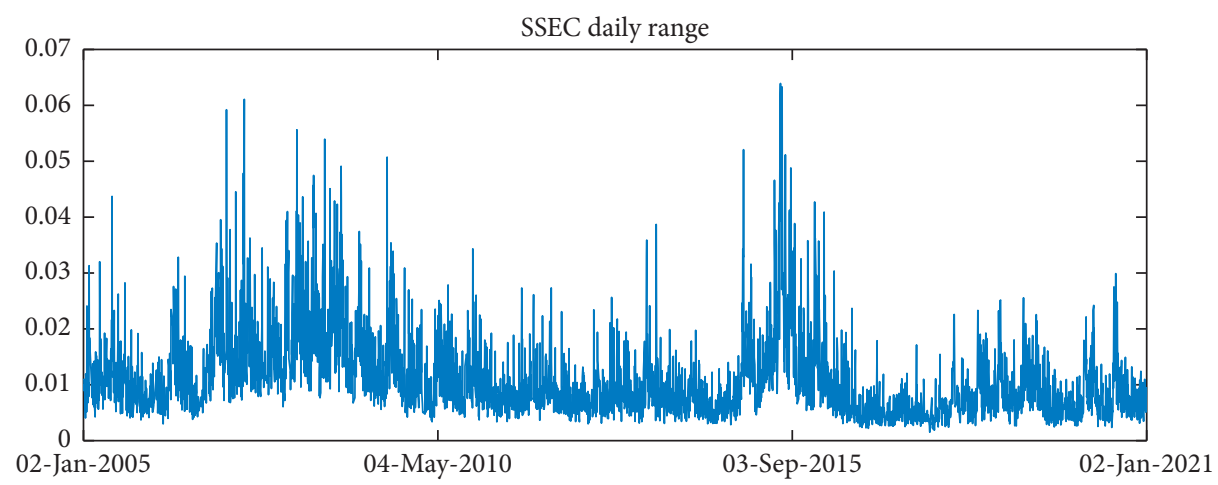

(a)

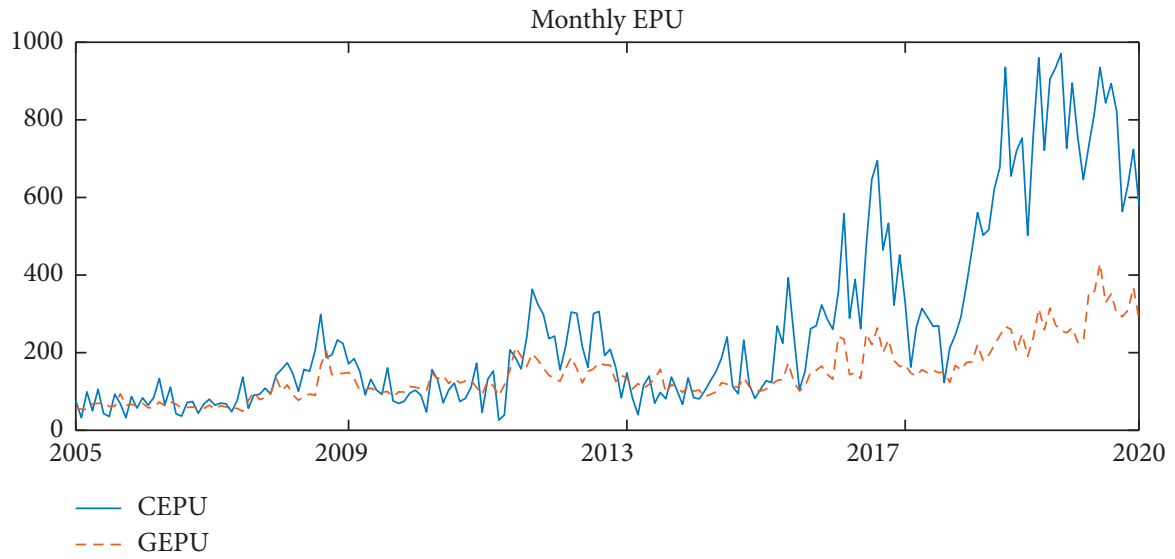

(b)

FIGURE 1: Daily range of SSEC and monthly EPU (CEPU and GEPU).

TABLE 1: Summary statistics.

\begin{tabular}{|c|c|c|c|c|c|c|c|c|}
\hline & Mean & Min. & Max. & Std. dev. & Skewness & Kurtosis & Jarque-Bera & $\mathrm{Q}(10)$ \\
\hline \multicolumn{9}{|c|}{ Panel A: daily range } \\
\hline SSEC & 0.0110 & 0.0015 & 0.0639 & 0.0075 & 2.1969 & 9.9680 & 10995.7980 & 9176.8674 \\
\hline \multicolumn{9}{|c|}{ Panel B: monthly EPU } \\
\hline CEPU & 261.6636 & 26.1441 & 970.8299 & 242.6354 & 1.4383 & 4.0095 & 74.3495 & 1240.5849 \\
\hline GEPU & 145.2330 & 48.8196 & 429.5147 & 72.9342 & 1.2406 & 4.4544 & 66.1778 & 1015.1523 \\
\hline
\end{tabular}

$\mathrm{Q}(10)$ is the Ljung-Box statistic for autocorrelation up to 10 lags.

TABle 2: Estimation results.

\begin{tabular}{lcccc}
\hline & CARR & CARR-MIDAS & CARR-MIDAS-CEPU & CARR-MIDAS-GEPU \\
\hline$\alpha$ & $0.1532(0.0075)$ & $0.1739(0.0024)$ & $0.1731(0.0024)$ & $0.1726(0.0024)$ \\
$\beta$ & $0.8340(0.0080)$ & $0.7705(0.0027)$ & $0.7677(0.0028)$ & $0.7679(0.0028)$ \\
$m(\omega)$ & $0.0001(0.0000)$ & $-2.1709(0.0060)$ & $-1.8975(0.0053)$ & $-1.5643(0.0045)$ \\
$\theta_{1}$ & & $0.3976(0.0020)$ & $0.3456(0.0024)$ & $0.3506(0.0023)$ \\
$\gamma_{1}$ & $11.1079(0.0544)$ & $12.3186(0.0061)$ & $11.7892(0.0721)$ \\
$\theta_{2}$ & & $-0.1172(0.0027)$ & $-0.1854(0.0027)$ \\
$\gamma_{2}$ & & & $1.9172(0.0122)$ & $7.3200(0.0405)$ \\
$\nu$ & & $5.6324(0.0092)$ & $5.6420(0.0096)$ & $5.6443(0.0087)$ \\
Log-lik & $5.6010(0.1266)$ & 15981.5230 & 15985.0211 & 15985.8600 \\
AIC & 15970.0035 & -31951.0460 & -31954.0421 & -31955.7200 \\
\hline
\end{tabular}

Log-lik is the log-likelihood, and AIC is the Akaike information criterion. Numbers in parentheses are standard errors for the model parameters. 


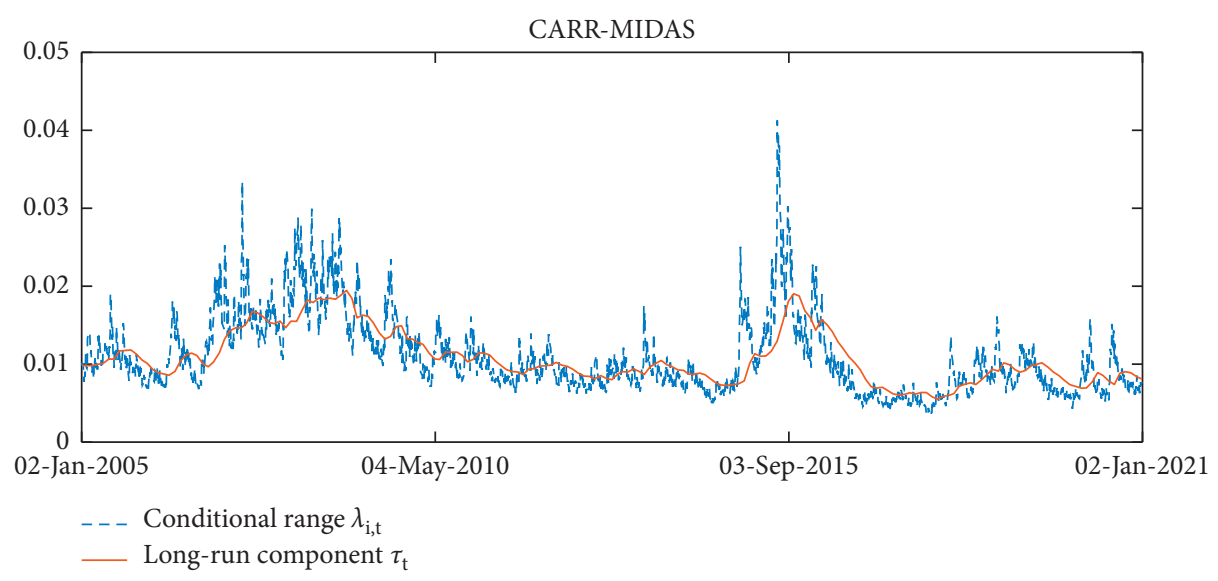

(a)

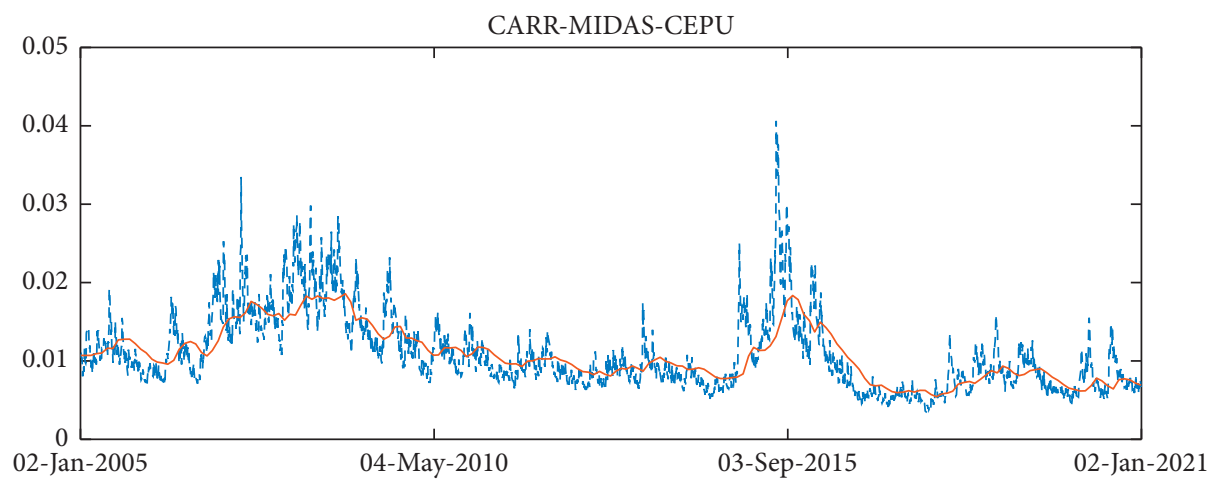

- - - Conditional range $\lambda_{\mathrm{i}, \mathrm{t}}$

_ Long-run component $\tau_{\mathrm{t}}$

(b)

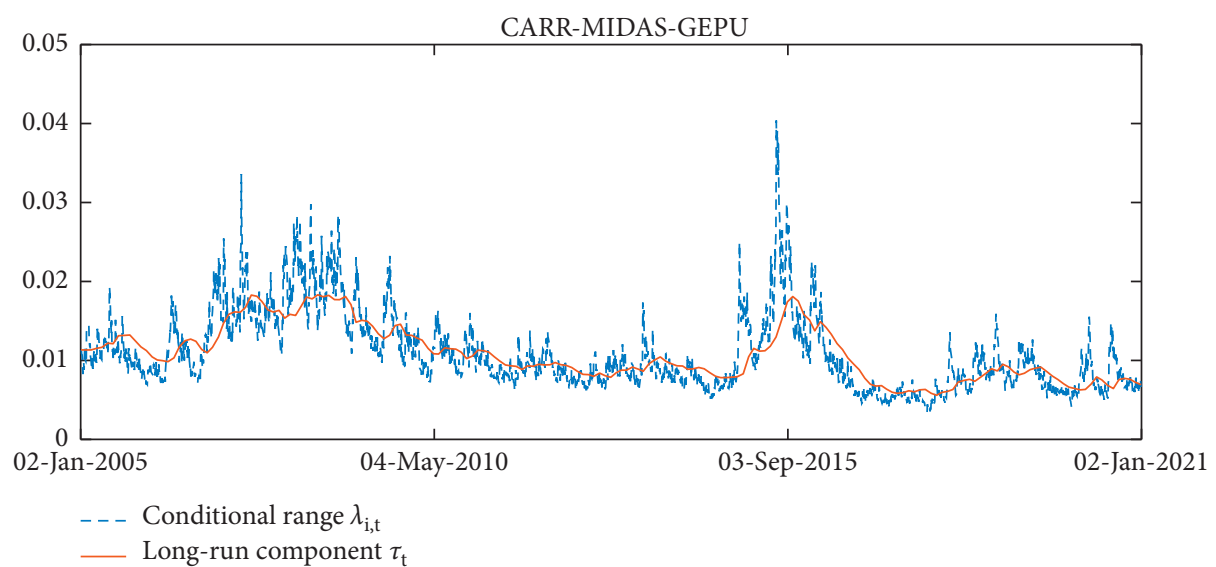

(c)

Figure 2: Conditional range $\left(\lambda_{i, t}\right)$ and the long-run component $\left(\tau_{t}\right)$ from the CARR-MIDAS framework.

framework. It is obvious that the long-run component of volatility appears smooth and tracks secular volatility trends over the sample period. All of the CARR-MIDAS models (the standard CARR-MIDAS, the CARR-MIDAS-CEPU, and the CARR-MIDAS-GEPU) do a good job in capturing the long-run trend of Chinese stock market volatility.

Turning to the shape parameter of the Gamma distribution, $v$ 's in the models are estimated to be significantly larger than one, ranging from 5.60 to 5.65. The estimates of $v$ indicate a significant deviation from the exponential distribution.

Finally, we find that the standard CARR-MIDAS model significantly improves the CARR model, in terms of the loglikelihood and the Akaike information criterion (AIC). The result demonstrates that the multiplicative component structure for the conditional range is important to improve the model fit. Moreover, the extended CARR-MIDAS 
TABLE 3: Out-of-sample forecast evaluation results.

\begin{tabular}{|c|c|c|c|c|}
\hline Horizon & CARR & CARR-MIDAS & CARR-MIDAS-CEPU & CARR-MIDAS-GEPU \\
\hline \multicolumn{5}{|c|}{ Panel A: MSE loss function } \\
\hline 1 & $6.4698 e-06$ & $5.6615 e-06$ & $4.0326 e-06$ & $4.7452 e-06$ \\
\hline 5 & $8.7122 e-06$ & $7.4566 e-06$ & $4.9309 e-06$ & $5.9348 e-06$ \\
\hline 10 & $1.0675 e-05$ & $8.7959 e-06$ & $5.4236 e-06$ & $6.6263 e-06$ \\
\hline 22 & $1.3883 e-05$ & $9.8059 e-06$ & $5.5660 e-06$ & $6.7895 e-06$ \\
\hline \multicolumn{5}{|c|}{ Panel B: QLIKE loss function } \\
\hline 1 & $5.3383 e-02$ & $4.8970 e-02$ & $3.6363 e-02$ & $4.2160 e-02$ \\
\hline 5 & $6.9746 e-02$ & $6.4000 e-02$ & $4.5982 e-02$ & $5.3552 e-02$ \\
\hline 10 & $8.3782 e-02$ & $7.4541 e-02$ & $5.1807 e-02$ & $6.0427 e-02$ \\
\hline 22 & $1.0811 e-01$ & $8.5781 e-02$ & $5.8370 e-02$ & $6.6381 e-02$ \\
\hline
\end{tabular}

MSE is the mean squared error, and QLIKE is the quasilikelihood. Bold entries indicate the model with the lowest loss value per horizon (in each row). Shaded entries indicate the model is included in the MCS at a $10 \%$ significance level.

models (CARR-MIDAS-CEPU and CARR-MIDAS-GEPU) outperform the standard CARR-MIDAS model, which highlights the value of incorporating EPU into range (volatility) modelling. Note also that the CARR-MIDAS-GEPU model provides a slightly better performance in empirical fit than the CARR-MIDAS-CEPU model.

3.3. Out-of-Sample Results. In this section, we investigate whether EPU has predictive ability on Chinese stock market volatility using the CARR-MIDAS approach. The outof-sample exercise is performed based on a rolling window (parameter estimates) scheme with a fixed window size of 3000 trading days. For each new forecast, we roll the window forward daily. The forecast horizon is set to one day, one week, two weeks, and one month, i.e., 1-day, 5-days, 10-days, and 22-days ahead forecasts.

As volatility cannot be observed directly, we require a proxy of the true volatility for forecast evaluation. In this paper, we use the daily realized range volatility (RRV) based on 5-min high-frequency data as the proxy of the true volatility, which is defined as

$$
\operatorname{RRV}_{i, t}=\sum_{j=1}^{J} R_{j, i, t}^{2},
$$

where $R_{j, i, t}$ is the $j$ th intraday range at day $i$ in month $t$. It has been documented in the literature that RRV is five times more efficient than the realized volatility measure (see, e.g., Christensen and Podolskij [33]).

To assess the forecast performance of the CARR-MIDAS approach, we use two robust loss functions, the mean squared error (MSE) and the quasilikelihood (QLIKE), which are given by

$$
\begin{aligned}
\text { MSE: } \operatorname{loss}_{i, t} & =\left(\mathrm{MV}_{i, t}-\mathrm{FV}_{i, t}\right)^{2}, \\
\text { QLIKE: } \operatorname{loss}_{i, t} & =\frac{\mathrm{MV}_{i, t}}{\mathrm{FV}_{i, t}}-\log \frac{\mathrm{MV}_{i, t}}{\mathrm{FV}_{i, t}}-1,
\end{aligned}
$$

where $\mathrm{MV}_{i, t}=\sqrt{\mathrm{RRV}_{i, t}}$ is the measured volatility (Since RRV measures the variance (squared volatility), we take the square root transformation for RRV as the measured (true) volatility.) and $F V_{i, t}$ is the forecasted volatility. Patton [34] showed that the MSE and QLIKE loss functions are robust to imperfect volatility proxies and provide consistent ranking of forecasts.

Moreover, we evaluate the predictive ability of the CARR-MIDAS approach by employing the model confidence set (MCS) test of Hansen et al. [35]. The MCS procedure examines a given set of competing models and identifies the set of the best performing models with some confidence level, namely, the MCS. This procedure is performed based on an equivalence test and an elimination rule. To be specific, let $\mathscr{M}^{0}$ be an initial set of all competing models. The null hypothesis for the equivalence test is given by

$$
H_{0, \mathscr{M}}: E\left(d_{u v, i, t}\right)=0, \quad \forall u, v \in \mathscr{M}, \mathscr{M} \subset \mathscr{M}^{0},
$$

where $d_{u v, i, t} \equiv \operatorname{loss}_{i, t}(u)-\operatorname{loss}_{i, t}(v)$ denotes the difference in the MSE or QLIKE loss of models $u$ and $v$. Following Hansen et al. [35], the test statistic for the null hypothesis $H_{0, \mathscr{M}}$ is given by

$$
\begin{aligned}
T_{\mathscr{M}} & =\max _{u, v \in \mathscr{M}}\left|t_{u v}\right|, \\
t_{u v} & =\frac{\bar{d}_{u v}}{\sqrt{\widehat{\operatorname{var}}\left(\bar{d}_{u v}\right)}},
\end{aligned}
$$

where $\bar{d}_{u v}$ is the average loss difference and $\widehat{\operatorname{var}}\left(\bar{d}_{u v}\right)$ denotes a bootstrapped estimate of $\operatorname{var}\left(\bar{d}_{u v}\right)$. If the null hypothesis $H_{0, \mathscr{M}}$ is rejected, the set $\mathscr{M}$ is reduced using the elimination rule, $e_{\mathscr{M}}=\operatorname{argmax}_{u \in \mathscr{M}} \sup _{v \in \mathscr{M}} t_{u v}$. The test is performed iteratively, until no further model can be eliminated. The final set of surviving models are denoted by $\mathscr{M}_{M C S}$. Following Hansen et al. [35], we implement the MCS procedure using a block bootstrap of $10^{5}$ replications and a significance level of $10 \%$.

Table 3 presents the out-of-sample forecast evaluation results. It can be seen from the table that the standard CARR-MIDAS model improves upon the forecasting performance of the CARR model for all forecast horizons in terms of the two loss functions. This indicates the usefulness of modelling a long-run component (or multiplicative component structure). More importantly, by incorporating 
TABLE 4: Out-of-sample forecast evaluation results for forecast window of 500.

\begin{tabular}{|c|c|c|c|c|}
\hline Horizon & CARR & CARR-MIDAS & CARR-MIDAS-CEPU & CARR-MIDAS-GEPU \\
\hline \multicolumn{5}{|c|}{ Panel A: MSE loss function } \\
\hline 1 & $5.8635 e-06$ & $5.3300 e-06$ & $3.9646 e-06$ & $4.2818 e-06$ \\
\hline 5 & $8.0498 e-06$ & $7.2398 e-06$ & $5.0020 e-06$ & $5.4611 e-06$ \\
\hline 10 & $9.9444 e-06$ & $8.7142 e-06$ & $5.6484 e-06$ & $6.2006 e-06$ \\
\hline 22 & $1.3656 e-05$ & $1.0430 e-05$ & $6.3693 e-06$ & $6.9867 e-06$ \\
\hline \multicolumn{5}{|c|}{ Panel B: QLIKE loss function } \\
\hline 1 & $4.6432 e-02$ & $4.5667 e-02$ & $3.4571 e-02$ & $3.7338 e-02$ \\
\hline 5 & $6.1724 e-02$ & $6.1738 e-02$ & $4.4787 e-02$ & $4.8592 e-02$ \\
\hline 10 & $7.4483 e-02$ & $7.3153 e-02$ & $5.1065 e-02$ & $5.5565 e-02$ \\
\hline 22 & $1.0084 e-01$ & $8.8528 e-02$ & $6.1062 e-02$ & $6.5505 e-02$ \\
\hline
\end{tabular}

MSE is the mean squared error, and QLIKE is the quasilikelihood. Bold entries indicate the model with the lowest loss value per horizon (in each row). Shaded entries indicate the model is included in the MCS at a $10 \%$ significance level.

TABLE 5: Out-of-sample forecast evaluation results for forecast window of 1000 .

\begin{tabular}{|c|c|c|c|c|}
\hline Horizon & CARR & CARR-MIDAS & CARR-MIDAS-CEPU & CARR-MIDAS-GEPU \\
\hline \multicolumn{5}{|c|}{ Panel A: MSE loss function } \\
\hline 1 & $6.3291 e-06$ & $5.4761 e-06$ & $3.8849 e-06$ & $4.5401 e-06$ \\
\hline 5 & $8.6110 e-06$ & $7.2356 e-06$ & $4.7254 e-06$ & $5.6592 e-06$ \\
\hline 10 & $1.0671 e-05$ & $8.5469 e-06$ & $5.1621 e-06$ & $6.2875 e-06$ \\
\hline 22 & $1.4443 e-05$ & $9.6989 e-06$ & $5.2976 e-06$ & $6.4651 e-06$ \\
\hline \multicolumn{5}{|c|}{ Panel B: QLIKE loss function } \\
\hline 1 & $5.6089 e-02$ & $5.0384 e-02$ & $3.7588 e-02$ & $4.2935 e-02$ \\
\hline 5 & $7.3891 e-02$ & $6.5760 e-02$ & $4.6985 e-02$ & $5.4085 e-02$ \\
\hline 10 & $8.9510 e-02$ & $7.6431 e-02$ & $5.2300 e-02$ & $6.0477 e-02$ \\
\hline 22 & $1.1905 e-01$ & $8.9079 e-02$ & $5.8530 e-02$ & $6.6474 e-02$ \\
\hline
\end{tabular}

MSE is the mean squared error, and QLIKE is the quasilikelihood. Bold entries indicate the model with the lowest loss value per horizon (in each row). Shaded entries indicate the model is included in the MCS at a $10 \%$ significance level.

C(G)EPU, the extended CARR-MIDAS model, the CARRMIDAS-C(G)EPU model, provides better performance than the standard CARR-MIDAS model, suggesting that volatility forecasting can be improved when the $\mathrm{C}(\mathrm{G}) \mathrm{EPU}$ is taken into consideration. It is interesting to note the CARR-MIDAS-CEPU model outperforms the CARR-MIDAS-GEPU model in all cases. The results demonstrate that the CEPU is more important than the GEPU for forecasting volatility of Chinese stock market. Overall, the CARR-MIDAS-CEPU model consistently gives the lowest loss values and is clearly the preferred and best forecasting model, followed by the CARR-MIDAS-GEPU model.

The shaded entries in Table 3 identify the model included in the MCS at the significance level of $10 \%$. The results show that the CARR-MIDAS-CEPU model is the only model that includes in the MCS for all forecast horizons, suggesting that the CARR-MIDAS-CEPU model significantly outperforms all other models.

3.4. Robustness Check. For the robustness check, out-ofsample forecast is also performed on the SSEC over different forecast windows (out-of-sample periods). We choose two different forecast windows, 500 and 1000. The out-of-sample forecast evaluation results are reported in Tables 4 and 5 for the two forecast windows, respectively. As is consistent with the results in Table 3, the CARR-MIDAS-CEPU model performs significantly better than the others.

\section{Conclusions}

Motivated by the return-based GARCH-MIDAS model, we introduce in this paper the range-based CARR-MIDAS model that exploits intraday information from the high and low prices. The proposed CARR-MIDAS model allows for exogenous explanatory variable such as EPU to model the time-varying long-run volatility. We employ the CARRMIDAS approach to investigate the impact of EPU on the volatility of Chinese stock market and to explore the predictive ability of EPU for Chinese stock market. We perform an analysis on the SSEC of China and use two EPU indices including CEPU and GEPU. We find that both the CEPU and GEPU have a significantly negative effect on the longrun volatility of Chinese stock market. The multiplicative component MIDAS structure for the conditional range is important to improve the model fit. The CARR-MIDAS models significantly improve upon the empirical fit of the original CARR model. Moreover, the extended CARRMIDAS models (CARR-MIDAS-CEPU and CARR-MIDASGEPU) outperform the standard CARR-MIDAS model, which highlights the value of incorporating EPU into range (volatility) modelling. Furthermore, out-of-sample results demonstrate that taking into account the CEPU and GEPU substantially improves our ability to forecast 1-day, 5-days (one week), 10-days (two weeks), and 22-days (one month) ahead volatility of Chinese stock market. In particular, we find that the CEPU provides superior volatility forecasts 
compared to the GEPU. According to the robustness check, our empirical results are robust to different forecasting windows. Overall, the findings highlight the role of EPU particularly the CEPU as predictor of Chinese stock market volatility.

Finally, future research could be extended to consider application of our approach to risk management, or to option pricing relying on EPU. Moreover, our proposed model could be extended to incorporate regime switching in the short-run component of volatility in the spirit of the regime switching GARCH-MIDAS model of Pan et al. [36]. The regime switching model has the capacity to accommodate structural breaks, which is a well-known stylized feature of financial volatility.

\section{Data Availability}

The data on the SSEC index of China are obtained from Wind Database of China, and the data on the monthly CEPU and GEPU indices are obtained from https://www.policy. uncertainty.com/. All the data are available from the corresponding author upon request.

\section{Conflicts of Interest}

The authors declare that there are no conflicts of interest regarding the publication of this paper.

\section{Acknowledgments}

This research was supported by the National Natural Science Foundation of China (No. 71971001), the University Natural Science Research Project of Anhui Province (No. KJ2019A0659), and the Innovative Research Project for Graduates of Anhui University of Finance and Economics (No. ACYC2020186).

\section{References}

[1] S. R. Baker, N. Bloom, and S. J. Davis, "Measuring economic policy uncertainty," Quarterly Journal of Economics, vol. 131, no. 4, pp. 1593-1636, 2016.

[2] S. A. Al-Thaqeb and B. G. Algharabali, "Economic policy uncertainty: a literature review," The Journal of Economic Asymmetries, vol. 20, Article ID e00133, 2019.

[3] L. Pástor and P. Veronesi, "Uncertainty about government policy and stock prices," The Journal of Finance, vol. 67, no. 4, pp. 1219-1264, 2012.

[4] Ľ. Pástor and P. Veronesi, "Political uncertainty and risk premia," Journal of Financial Economics, vol. 110, no. 3, pp. 520-545, 2013.

[5] L. Liu and T. Zhang, "Economic policy uncertainty and stock market volatility," Finance Research Letters, vol. 15, pp. 99105, 2015.

[6] M. Arouri, C. Estay, C. Rault, and D. Roubaud, "Economic policy uncertainty and stock markets: long-run evidence from the US," Finance Research Letters, vol. 18, pp. 136-141, 2016.

[7] Z. Liu, Y. Ye, F. Ma, and J. Liu, "Can economic policy uncertainty help to forecast the volatility: a multifractal perspective," Physica A: Statistical Mechanics and Its Applications, vol. 482, pp. 181-188, 2017.
[8] I.-C. Tsai, “The source of global stock market risk: a viewpoint of economic policy uncertainty," Economic Modelling, vol. 60, pp. 122-131, 2017.

[9] Y. Duan, W. Chen, Q. Zeng, and Z. Liu, "Leverage effect, economic policy uncertainty and realized volatility with regime switching," Physica A: Statistical Mechanics and Its Applications, vol. 493, pp. 148-154, 2018.

[10] D. Mei, Q. Zeng, Y. Zhang, and W. Hou, "Does US economic policy uncertainty matter for European stock markets volatility?" Physica A: Statistical Mechanics and Its Applications, vol. 512, pp. 215-221, 2018.

[11] X. Xiong, Y. Bian, and D. Shen, "The time-varying correlation between policy uncertainty and stock returns: evidence from China," Physica A: Statistical Mechanics and Its Applications, vol. 499, pp. 413-419, 2018.

[12] M. Yu and J. Song, "Volatility forecasting: global economic policy uncertainty and regime switching," Physica A: Statistical Mechanics and Its Applications, vol. 511, pp. 316-323, 2018.

[13] M. Balcilar, R. Gupta, W. J. Kim, and C. Kyei, "The role of economic policy uncertainties in predicting stock returns and their volatility for Hong Kong, Malaysia and South Korea," International Review of Economics \& Finance, vol. 59, pp. 150-163, 2019.

[14] T. C. Chiang, "Economic policy uncertainty, risk and stock returns: evidence from G7 stock markets," Finance Research Letters, vol. 29, pp. 41-49, 2019.

[15] R. F. Engle, E. Ghysels, and B. Sohn, "Stock market volatility and macroeconomic fundamentals," The Review of Economics and Statistics, vol. 95, no. 3, pp. 776-797, 2013.

[16] H. Yu, L. Fang, and W. Sun, "Forecasting performance of global economic policy uncertainty for volatility of Chinese stock market," Physica A: Statistical Mechanics and Its Applications, vol. 505, pp. 931-940, 2018.

[17] X. Yu and Y. Huang, "The impact of economic policy uncertainty on stock volatility: evidence from GARCH-MIDAS approach," Physica A, vol. 570, Article ID 125794, 2021.

[18] T. Li, F. Ma, X. Zhang, and Y. Zhang, "Economic policy uncertainty and the Chinese stock market volatility: novel evidence," Economic Modelling, vol. 87, pp. 24-33, 2020.

[19] X. Wang, Y. Luo, Z. Wang, Y. Xu, and C. Wu, "The impact of economic policy uncertainty on volatility of China's financial stocks: An empirical analysis," Finance Research Letters, Forthcoming.

[20] Y. Li, F. Ma, Y. Zhang, and Z. Xiao, "Economic policy uncertainty and the Chinese stock market volatility: new evidence," Applied Economics, vol. 51, no. 49, pp. 5398-5410, 2019.

[21] M. Parkinson, "The extreme value method for estimating the variance of the rate of return," Journal of Business, vol. 53, no. 1, pp. 61-65, 1980.

[22] S. Alizadeh, M. W. Brandt, and F. X. Diebold, "Range-based estimation of stochastic volatility models," The Journal of Finance, vol. 57, no. 3, pp. 1047-1091, 2002.

[23] M. W. Brandt and C. S. Jones, "Volatility forecasting with range-based EGARCH models," Journal of Business \& Economic Statistics, vol. 24, no. 4, pp. 470-486, 2006.

[24] H. Li and Y. Hong, "Financial volatility forecasting with range-based autoregressive volatility model," Finance Research Letters, vol. 8, no. 2, pp. 69-76, 2011.

[25] S. Degiannakis and A. Livada, "Realized volatility or price range: evidence from a discrete simulation of the continuous time diffusion process," Economic Modelling, vol. 30, pp. 212-216, 2013. 
[26] R. Y. Chou, H. Chou, and N. Liu, "Range volatility: a review of models and empirical studies," in Handbook of Financial Econometrics and Statistics, C.-F. Lee and J. Lee, Eds., Springer, New York, NY, USA, pp. 2029-2050, 2015.

[27] R. Y. Chou, "Forecasting financial volatilities with extreme values: the conditional autoregressive range (CARR) model," Journal of Money, Credit, and Banking, vol. 37, no. 3, pp. 561-582, 2005.

[28] F. Wang and E. Ghysels, "Econometric analysis of volatility component models," Econometric Theory, vol. 31, no. 2, pp. 362-393, 2015.

[29] C. Conrad and O. Kleen, "Two are better than one: volatility forecasting using multiplicative component GARCH-MIDAS models," Journal of Applied Econometrics, vol. 35, no. 1, pp. 19-45, 2020.

[30] H. B. Xie, "Range-based volatility forecasting: a multiplicative component conditional autoregressive range model," Journal of Risk, vol. 22, no. 5, pp. 1-23, 2020.

[31] C. G. Lamoureux and W. D. Lastrapes, "Persistence in variance, structural change, and the GARCH model," Journal of Business \& Economic Statistics, vol. 8, no. 2, pp. 225-234, 1990.

[32] H. Xie and X. Wu, "A conditional autoregressive range model with gamma distribution for financial volatility modelling," Economic Modelling, vol. 64, pp. 349-356, 2017.

[33] K. Christensen and M. Podolskij, "Realized range-based estimation of integrated variance," Journal of Econometrics, vol. 141, no. 2, pp. 323-349, 2007.

[34] A. J. Patton, "Volatility forecast comparison using imperfect volatility proxies," Journal of Econometrics, vol. 160, no. 1, pp. 246-256, 2011.

[35] P. R. Hansen, A. Lunde, and J. M. Nason, "The model confidence set," Econometrica, vol. 79, no. 2, pp. 453-497, 2011.

[36] Z. Pan, Y. Wang, C. Wu, and L. Yin, "Oil price volatility and macroeconomic fundamentals: a regime switching GARCHMIDAS model," Journal of Empirical Finance, vol. 43, pp. 130-142, 2017. 